\title{
SMSpress: An SMS Research Management System
}

\author{
Rushil Khurana, Yi Han, \& Rosa I. Arriaga \\ School of Interactive Computing \\ Georgia Institute of Technology \\ United States \\ \{rushil.khurana,yihan\}@gatech.edu, arriaga@cc.gatech.edu
}

\begin{abstract}
Text messaging is a functionality that has been widely adopted in user studies due to its presence in smart phones and feature phones alike. However the ability to use cell phones for data collection has been limited to researchers that have programming abilities. We present SMSpress, a web-based SMS management system that allows non-technical users to configure SMS studies. Researchers can schedule text messages for delivery to participants via a web-interface. We establish the requirements for such a system, and present the results from two case studies. One is a four month mhealth deployment with cardiac patients; the other is a usability evaluation of a study to help parents keep track of their child's development.
\end{abstract}

Keywords-ubicomp; research studies; web-based SMS

\section{INTRODUCTION}

There has been a significant increase in cell phone usage in the past decade. As of May 2013, $91 \%$ of adults in the U.S. have a mobile phone, smart phone or otherwise [1]. The popularity of mobile phones makes them an important tool in research studies. Reality Mining: Sensing Complex Systems, arguably one of the largest ubiquitous computing research studies used mobile phones as a medium to collect data for over 9 months [2].

While there has been a steady rise in smart phone ownership 35\% of American adults still own a feature phone [1]. Excluding non-smart phone users constitutes losing a substantial pool of potential research participants. This makes short messaging system (SMS, i.e. texting) an important tool for researcher since it is available in all cell phones. Further, its use allows researchers to target individuals from diverse socioeconomic backgrounds. Also, in certain situations, smartphones, internet and data plans are not easily available. SMS has a wider coverage and its delay-tolerance and P2P message passing properties also make it a suitable and lucrative alternative in such situations.

SMS studies are common in the HCI field. They have proven to be an important tool for researchers interested in all aspects of user behavior [2, 3, 4, 5]. However, it has thus far only been available for researchers that have programming abilities or access to development staff. There are a number of other researchers (i.e., psychologists, healthcare professionals, etc.) that can benefit from studies that leverage SMS. However they are stymied because the setup of such studies entails technical work, which in most cases is not their area of expertise. The economic cost of hiring an individual to handle the technical setup can also be a deterrent. In this paper, we present SMSpress a web-based SMS research management system that can be used by non-programmers. It enables researchers with no programming skills to customize and manage SMS studies.

\section{RELATED WORK}

SMS studies have emerged in a range of fields from HCI to healthcare. Experience sampling has been one methodology that has taken advantage of the SMS platform. Andrews, Bennet and Drennan used SMS to collect measures of consumers' experiences in a method called Experience Sampling [15]. Markett et al. introduced an SMS system to students to make the classroom interactive [16]. In the behavior modification area, Rodgers et al. conducted a study to demonstrate SMS intervention is an effective smoking cessation tool [7]. While Free et al. showed that text interventions can help improve continuous abstinence from smoking [9]. With the increase cell phone use among children researchers have also targeted pediatric patients. Researchers have deployed intervention for chidren with diabetes $[8,10]$ and asthma [4,11].

There are several tools that can collect data from cell phones via text messaging. One such popular framework is RapidSMS. RapidSMS has been used as a health information collection tool in a variety of domains [12, 13, 14]. Mendizabal compiled basic information about different mobile data collection and research tools [5]. Currently, these tools and frameworks entail some form of technical support for their customization. This poses a big constraint on research studies where the researchers lack technical expertise to leverage these tools. 


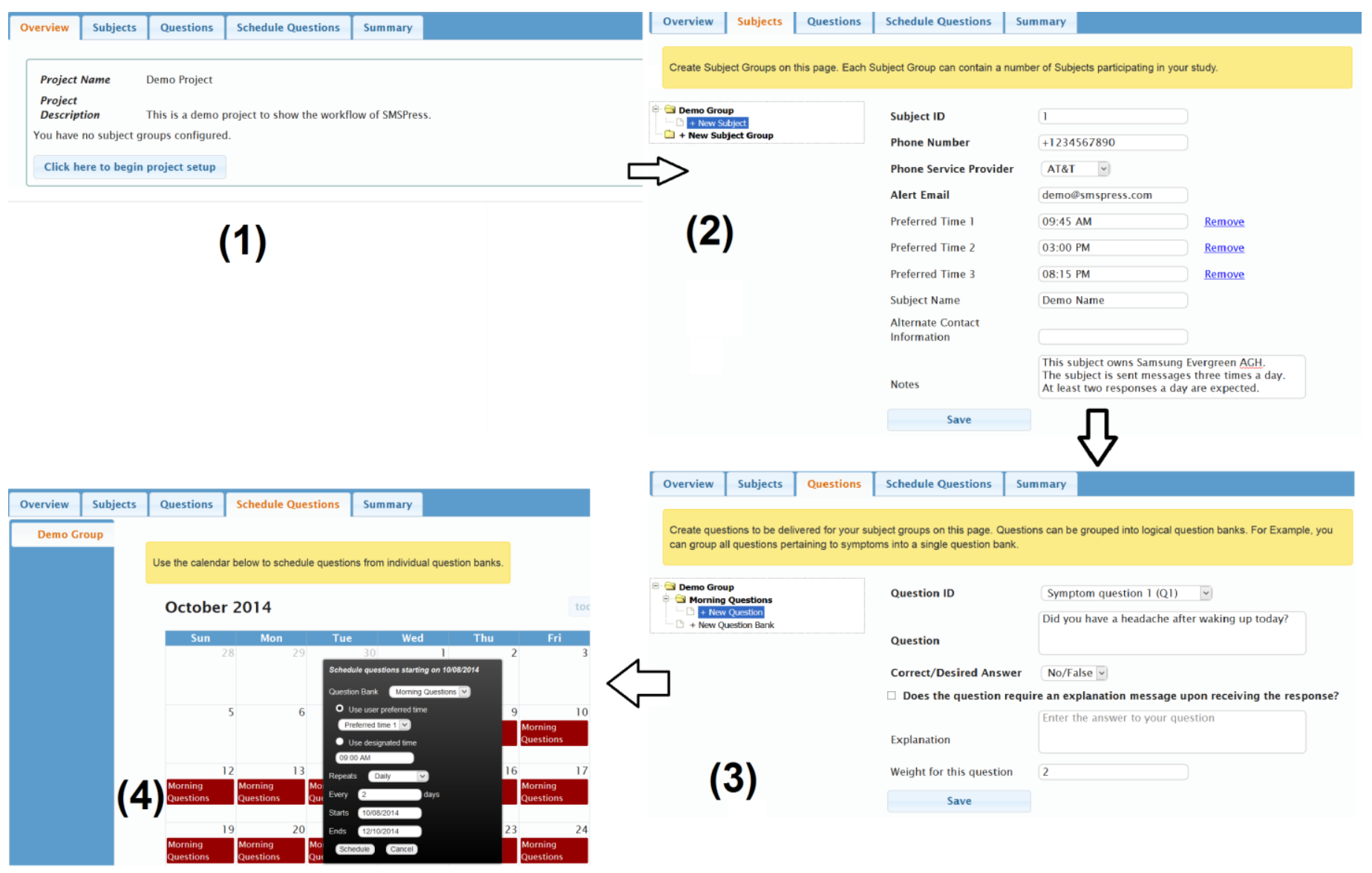

Figure 1: This is a workflow diagram that depicts how a project is configured starting with (1) the overview, (2) setting up a subject group, (3) configuring a question bank and (4) scheduling the question bank.

Momento was the first system to try to bridge the gap for non-technical researchers. The tool allowed researchers to communicate with the participants using the SMS/MMS feature present in phones. Alternatively, it allowed researchers to gather data from the sensors present in the cell phonelocation via GPS, proximity via Bluetooth, etc. However, Momento has a number of shortcomings that need to be addressed if SMS is to be used to its full capacity. First, the server side of the system does not communicate back with the participants of the study unless the researcher manually wishes to do so. This concern was echoed by the participants in one of the studies. The participants expressed frustration due to lack of immediate feedback from the researchers. Thus, another feature that needs to be included in an SMS management system a set of alerts that engage the researcher when crucial information is conveyed by the participant. Also, Momento provided a very basic data visualization option for their data. Finally, while it is laudable that the researchers had a deployment study for 51 days $(\mathrm{N}=14)$ they did not provide data for the number of SMS messages sent out by the system.

The literature review shows that professionals, psychologists etc. often conduct user studies leveraging the text messaging service. Research shows that the potential benefits that can be reaped from such studies are compelling (smoking cessation, improved medication adherence). However the technical know-how required to leverage such system is a barrier for those without computing support. Likewise, hiring a technical person to take care of the technology requirements can be prohibitive. In order to address these issues as well as address some of the shortcomings in [6] we present SMSpress. It is a first step towards a robust SMS research management system. It provides easy configuration and customization to a researcher who possesses basic knowledge of computers (browser basics, using basic word processing and spreadsheet applications, common keyboard shortcuts etc.) without any advanced technical skills.

\section{SYSTEM DESCRIPTION}

\section{A. System Overview}

SMSpress allows non-technical researchers to deploy the basic research tool, the survey, in an SMS format. SMSpress has a customizable calendar where the researcher can decide when and what subject or group of subjects will receive the text messages. Finally, the researcher can assign weights to questions that will activate an alert from SMSpress to the researcher based on the response from participants. It allows for visualization of the data by accumulated weights or by temporal resolution. Researcher can also allow participants to decide when they want to receive their messages. 


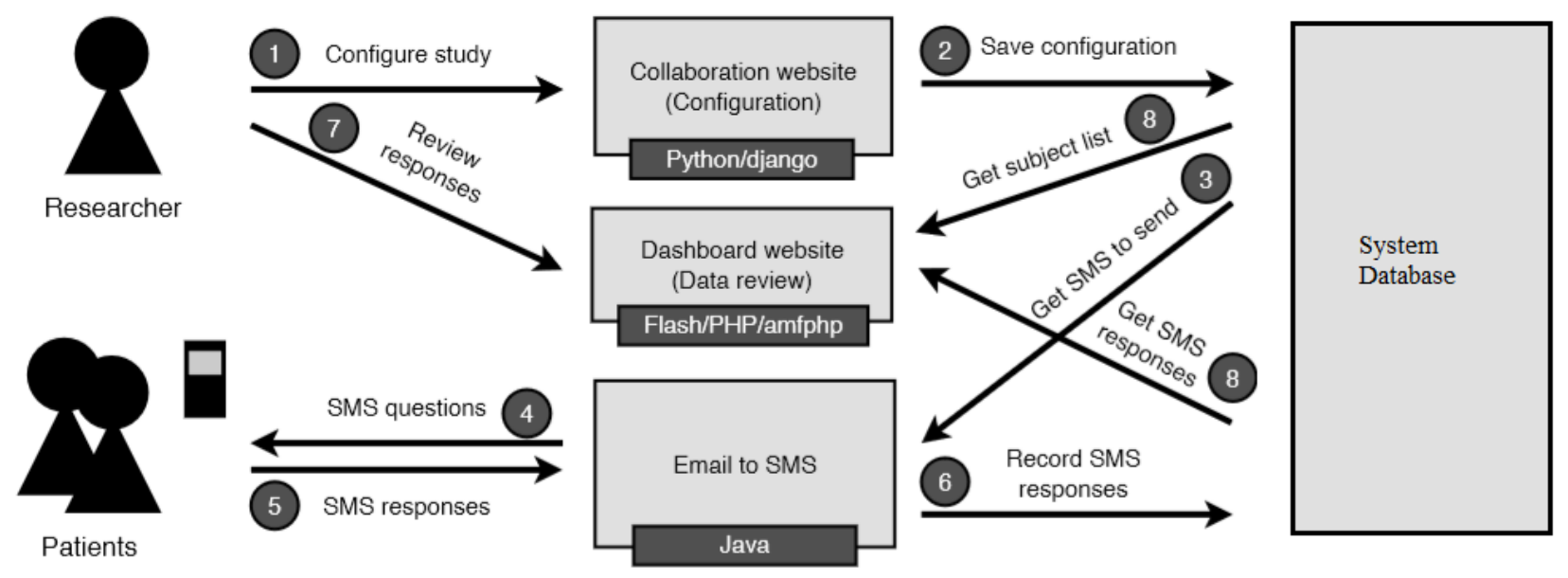

Figure 2: System architecture of SMSpress and the steps of communication between each component of the system

The typical research study can consist of multiple subject groups. Each subject group can have multiple participants. Participants are delivered information via SMS which may or may not involve communicating back with the server.

The current design maps well to the common use-case where all subjects in a particular group should receive the same SMS. Furthermore we group each SMS into message banks. This functionality allows the researcher to logically group related questions. An additional advantage is that the researcher can set a schedule for the whole message bank at once rather than individually scheduling each question. Also, it is a useful feature to have text messages delivered in varied schedules within a given subject group (say once versus multiple times a day). Thus, the ability to customize the schedules at the level of the message bank strikes a good balance between usability and flexibility.

\section{B. System Architecture}

The primary components of the SMSpress system are:

\section{1) Configuration Interface}

SMSpress has a web interface built on the Django framework that allows the researcher to configure the various settings (see Figure 1). The following information can be set on the configuration interface: subjects, SMS and question schedule.

\section{2) E-mail to SMS}

The system utilizes the email to SMS feature provided by the mobile phone service carriers to send and receive SMSes. We decided to use the E-mail to SMS feature because using the e-mail aliasing allows us to map the responses of a study participant with the correct SMS over a period of time. This is irrespective of whether the participant experienced delay in receiving messages or received them out of order. Each SMS can be associated with a different alias.

This feature also allows the researchers to send and receive SMS for free over email. Using the e-mail to SMS feature has a monetary cost associated with internet connectivity, which in most cases is borne by the researcher's organization.
In real world studies, participants have different mobile carriers. This puts a strain on the approach we used because every service provider handles the email-to-SMS functionality a little differently. Therefore, to use this feature, we had to find the greatest common message syntax that the different e-mail to SMS services supports. The e-mail to SMS program periodically checks the database of scheduled questions. It does this to query the schedule which is further associated with the text message and the participant in the database. If it is time to send a text message, it will send it to the intended participant(s).

When the participants respond to the text message with an SMS response, the response is received in the form of an email. The e-mail to SMS program will periodically check the inbox (currently 1 minute intervals) to capture the responses.

\section{3) System Database}

The database stores all the configurations i.e. subjects, questions and the delivery schedules that were setup via the configuration interface by different researchers. It also stores the SMS responses received via participants in the last step.

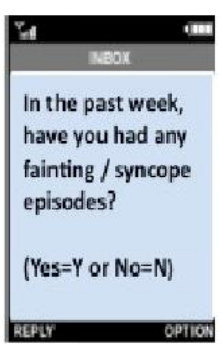

(A)

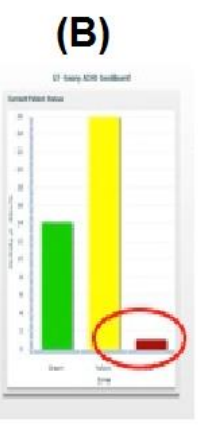

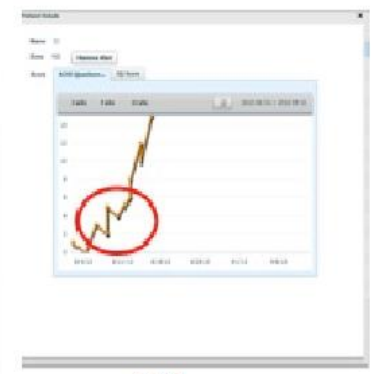

(C)
Figure 3: An example SMS (A) and visualization snapshots of both accumulated weighted responses $(B)$ and temporal resolution of the data $(\mathrm{C})$. 
TABLE I. OVERVIEW OF SMSPRESS FUNCTIONALITY BEING USED BY EACH RESEARCH STUDY

\begin{tabular}{|l|c|c|}
\hline SMSPress Features & Congenital Heart Disease & Milestone Tracking \\
\hline \multicolumn{3}{|c|}{ User Management Features } \\
\hline 1. Different Subject Groups & $\mathrm{X}$ & $\mathrm{X}$ \\
\hline $\begin{array}{l}\text { 2. Question banks based on subject } \\
\text { groups }\end{array}$ & $\mathrm{X}$ & $\mathrm{X}$ \\
\hline \multicolumn{3}{|c|}{ Content Delivery } \\
\hline 1. Scheduled SMSes & $\mathrm{X}$ & \\
\hline 2. Auto-system response & \multicolumn{1}{|c|}{ Data Management } \\
\hline \multicolumn{3}{|c|}{ X A. Study Setup } \\
\hline 1. Participant Responses Database & \multicolumn{1}{|c|}{ Xata Visualization } \\
\hline \multicolumn{1}{|c|}{ X } \\
\hline 1. Dashboard Interface
\end{tabular}

It is a web interface that visualizes the SMS responses received from the participants of the study. It pulls out data from both databases (scheduled questions and participant responses) to visualize and present to the researcher as shown in Figure 3. The web interface is built on Flash and PHP and was previously developed to visualize patient data for children with asthma [17].

Figure 2 shows the system architecture of SMSpress and the different steps of communication between the various components of the system. Figure 1 shows a workflow of configuring a project in the system.

\section{Methodology}

To understand the impact and usability of SMSpress as an SMS research management system, we evaluated it in two different research studies. Table 1 provides an overview of the functionalities provided by SMSpress in each of the research projects. The research studies, their setup and the researchers' feedback are described in detail in the following sections.

\section{STUDY 1- SMS INTERVENTION FOR CONGENITAL HEART DisEASE PATIENTS}

A nursing student used SMSpress for her graduate research. Her research goal was to determine the clinical relevance and feasibility of SMS as an intervention tool for enhancement of self-care, medical adherence and symptom awareness. Adults with congenital heart disease were the target population.

Since this was the first study on the SMSpress system, we offered passive technical assistance to the primary user i.e. we allowed the researcher to troubleshoot on her own initially, but assisted her if she was unable to fix the issue. The researcher was, a doctoral candidate in her 50's with no development skills and little experience with functions other than checking email, searching the web and some products in the Microsoft Office Suite.

\section{A. Study Setup}

The study was a randomized controlled trial with 60 participants divided into three groups ${ }^{1}$. Group 1 received a single message a day, group 2 received 2 to 3 messages a day, depending on the patient's medication schedule and the third group (control) received no SMSes. The study lasted 4 months. The setup of the study required different groups to receive different messages at varied times in the day. The configurations required for this particular project covered almost all the cases we would have liked to test on our system. Therefore, this study made for an ideal test bed.

The questions were also weighted into three categories based on the importance of information contained in them. If a patient answered affirmatively to a set of marked questions, then SMSpress would send an alert to the researcher. This demonstrates the usefulness of the weighted questions bank functionality of our system. Some of the questions were so important that they were flagged for alerting the researcher (e.g., did you pass out or faint in the last 4 weeks?).

\section{B. SMSpress Utilization Results}

Over the course of the study, a total of 4825 messages were delivered to participants and 3392 responses were received. The duration of the study and number of messages exchanged helped us test the robustness of the system. Table 2 shows a detailed summary of the number of messages sent by the system during the course of the study and the number of participant responses that were received.

\footnotetext{
${ }^{1}$ This research was conducted with institutional review board approval.
} 
TABLE II. SUMMARY OF MESSAGES SENT AND RESPONSES

\begin{tabular}{|l|l|l|l|}
\hline & $\begin{array}{l}\text { Messages } \\
\text { Sent }\end{array}$ & $\begin{array}{l}\text { Responses } \\
\text { Received }\end{array}$ & $\begin{array}{l}\text { Response } \\
\text { Rate (in \%) }\end{array}$ \\
\hline Month 1 & 1641 & 1211 & 73.79 \\
\hline Month 2 & 1619 & 1161 & 71.71 \\
\hline Month 3 & 1565 & 1020 & 65.17 \\
\hline
\end{tabular}

\section{Researcher's experience \& feedback}

The researcher was interested in a mhealth intervention for her dissertation work but had no technical support available to her. She noted that based on her exploration, SMSpress was the closest to what she needed in terms of functionality. She pointed out that the other options she explored were commercial apps and thus not suited for conducting user studies with sensitive data. She noted that she was afraid that requiring her participants to have a smart phone would make the recruiting more difficult. She also thought that it would skew the demographics of her study toward wealthier individuals that could afford data plans.

The researcher underwent two training sessions, which served as introductory lessons into the working of the system. The system was changed after the first session and another training session was required to introduce the researcher to the changes that had been made in the system. Since this was the first study, we offered the researcher assistance. However, after the two training sessions, the researcher was able to setup the intervention by herself. She also added herself to each group, so that she could monitor the SMSes that were being received by her patients. Thus, she could make sure that the three different groups were receiving the appropriate messages.

During the 4-month deployment period she noted that most of the groups received all of the intended messages. She was able to detect a few problems in the configurations she had made based on the texts she was receiving and feedback from the participants. There were three different kinds of problems encountered. Interestingly, these were all due to either 1) human error, on her part, when she set up the study; or 2) errors related to the mobile interface. The first type of problems included typos and misrepresentation of characters in a text message. She was able to fix most of these errors by herself, but asked for assistance for the other two when she was unable to determine their cause. The other two problems included bad numbering of one of the questions, which generated a conflict in the system; and choosing the wrong service provider for one of the participants. When she asked for assistance, we encouraged her to fix the problem herself in the configurations of the system. As soon as the cause was determined, she was able to access the system and fixed the problem herself.

During the initial weeks of the deployment the researcher informed us that the automatic alerts did not contain enough information. Thus we reconfigured the alerts. The alert message now includes the subject group that triggered the alert. This makes it easier for the researcher to quickly ascertain who requires assistance.
We collected feedback from the researcher, via a brief unstructured interview, during the last weeks of the deployment. This allowed us to capture reaction based on her long-term experience with the system i.e. both, initial usability experience and maintainability. She felt that the work of adding questions for each group separately was labor intensive, but the system was quite flexible in the functionality it provided. She pointed out that a basic troubleshooting manual would greatly enhance the usability of the system. The researcher concluded that she was very satisfied with SMSpress and that she could not have conducted her dissertation study without it. She also noted that she could easily setup another study without any help now.

\section{Study 2- Milestone Tracking System PRototype}

"Developmental milestones" refer to cognitive, behavioral, motor or social changes that occur in most children at a given age. For example, most children start walking around 12 months. The milestone tracking study was conducted by a 25 year old HCI researcher with a focus on psychology with little development skills. The aim of the study was to understand the familiarity of low socioeconomic status (SES) parents' with technology and their current practices to learn about children's milestones. The objective of the study was to gather insights for designing tracking systems for parents to monitor their child's development.

SMSpress was used to demonstrate the use of SMS service as a milestone tracking system, to the parents participating in the research study. SMS was compared against booklet, kiosk, mobile app and website as alternative milestone tracking systems. SMSpress was used to prototype the SMS alternative for qualitative evaluation. In the currently described study, it was used as a prototyping tool and not to evaluate the milestone system itself. The study is described in detail in the following sections.

\section{A. Study Setup}

SMSpress was used as a tool to Wizard of $\mathrm{Oz}$ a textmessage based milestone tracking system. This was used to gauge the participant's "in the moment" reactions. It was also used to understand their perceptions of such a system. The participants were either presented with an SMS question or factoid about children's development. The 50 SMSes were divided into different milestone categories based on children's age. The qualitative study was conducted with 21 participants.

The researcher (hereon referred to as "primary researcher") was interested in finding out the most suitable medium to serve as a milestone tracking system. He wanted to obtain user feedback without fully investing resources in the development of each possible alternative. Since the researcher was in the initial usability study phase he emphasized the need for rapid prototyping tools. The goal was to use SMSpress to emulate an ad-hoc SMS Q/A system which would generate specific messages based on the user's response to a given text message. 


\section{B. Researcher's experience and feedback}

Both the primary researcher and the research assistant were introduced to SMSpress and its functional capabilities, however were not provided a tutorial. The primary researcher conceptualized the configuration that allowed SMSpress to be used as a prototype of the SMS milestone tracking system. He then communicated this to the research assistant; an 18-yearold undergraduate with no prior development experience. The research assistant was able to configure the entire study by herself. SMSpress delivered the 50 milestone texts to the mobile phone that was used for the evaluation.

The primary researcher used the mobile phone pre-loaded with 50 SMSes as a prototype of a personalized SMS-based milestone tracking system. Each of the SMSes was configured to generate specific information based on a yes/no response from the users. The user evaluation consisted of asking the participants to imagine that the messages were personalized based on their child's age and to give feedback on their experience with the system.

We sought feedback from the research assistant right after she utilized SMSpress for the first time. We conducted a brief unstructured interview to capture her experience of interacting with the web system. She did not have any trouble with the task and found the system understandable and easy to use. She also appreciated the brief description provided for each functionality on top of its respective page in the system. She pointed out that she did not understand how to set up a response based on user interaction i.e. yes/no reply from the user, however was able to figure it out after initial exploration. We also conducted a semi-structured interview with the primary researcher after the end of his study to gauge his experience in using SMSpress as a quick prototype tool. He was satisfied with SMSpress' ability to emulate his envisioned system. He thought it was a quick and robust prototype-tool. He also expressed a desire to have the functionality of multiple system generated responses to the same initial SMS. Currently, SMSpress is restricted to one.

\section{LESSONS LEARNED}

The challenges involved in the development of an SMS research management system provided valuable lessons. One of the key takeaways is that when the web is used to interact with a user's mobile phone via SMS, each provider handles the e-mail to SMS functionality differently. Therefore, the system is required to be updated with the addition of a previously unknown service provider. Our special handling of the e-mail to SMS functionality works with a broad range of providers, however addition of a service provider needs to be tested before it can be rolled out. The e-mail to SMS service is required to keep track of messages sent to the participants and their responses. To the best of our knowledge, this is an improvement because traditional SMS gateway models require a modification to the delivery protocol in order to accomplish this task.

Another important lesson was that the architecture of the system should be evident in the web interface design. This allows the researcher to understand how the system works and use the given functionality in a way that best suits her needs.
This was evidenced in the second study where a researcher used SMSpress as a quick prototype tool (employing the Wizard of $\mathrm{Oz}$ technique). Prototyping was not an explicit usecase to drive the development of the system. This shows that when the primary user of the system (researcher) has a good mental model of the system he can leverage it in unexpected ways.

A final lesson learned is that a completely robust SMS management system like ours is impossible to achieve because addressing it is beyond any one researcher's ability. This problem is not unique to SMSpress it is the same for any system that leverages the e-mail to SMS functionality. We ensured that the system works with different service providers, however adding a new service provider requires testing that the user cannot do directly. The only way to completely eradicate this issue is to have the telecommunication industry standardizes the message syntax.

\section{DISCUSSION AND CONCLUSION}

SMS is increasingly being used as a research tool to conduct user studies. However, the technical know-how associated with setting up an SMS study becomes a barrier for researchers and professionals. SMSpress is an off-the-shelf system that can be used to setup SMS studies without requiring technical knowledge. We deployed SMSpress in two different studies and demonstrated its potential to aid researchers. Study 1 was a 4 month deployment study with 60 patients. In one group patients received up to 3 messages a day. SMSpress was found to be a robust system with no failures. Study 2 was a Wizard of Oz study. The second researcher needed a set of 50 different SMSes on his phone to successfully carry out his usability study. The researcher used SMSpress to schedule the delivery of the 50 questions on his phone. This simple task saved him the labor of sending 50 texts manually. This shows that the system was easy enough to understand that the researcher was able to come up with a novel use case for the system. Both of these studies were ecologically valid- the researchers actually needed a system to carry out their study and SMSpress met the requirements. Importantly to our claims about the ease-of-use of SMSpress, neither of the researchers required technical skills to set up their studies.

In both Study 1 and Study 2 researchers were highly satisfied with the results of SMSpress. In Study 1, the researcher reported that the errors were not technical in nature i.e. pertaining to development of the system, but due to human error during the setup. As HCI researchers, we aim to reduce such human errors as well and believe there is room for improving SMSpress. One improvement was made during the course of Study 1: we added subject group information to alerts. The other improvements we plan to implement will reduce the manual labor of adding questions by providing the option to upload questions from a document.

We were able to glean other improvements to SMSpress from the two studies; that were not obvious in our initial development. In the first study, an intervention, the researcher added herself to each of the groups to ensure reliability of the text delivery. In the future we can make this feature a default 
and have the researcher "opt out" of receiving messages. SMSpress can be extended in the future to allow such behavior by default with an option to opt-out, if needed. In the second study, a Wizard of Oz utilization of our system, the researcher expressed his desire to have multiple system-generated responses for a given initial question. Although, this functionality adds a layer of complexity to the existing system, we believe that it can widen SMSpress' scope of use.

In conclusion, researchers of both studies were quite satisfied with their overall experience and would like to use SMSpress in the future. This touts our tool as a promising first step towards building a robust SMS research management system. We also aim to make SMSpress available to a wider audience. The goal is to develop a single package installer that can setup the entire system with a single click. We believe that the system will allow and entirely new, non-technical, community to leverage the SMS functionality for research.

\section{ACKNOWLEDGMENT}

We thank Rohit Banga for his initial work on the development of SMSpress. We also thank the two researchers that used SMSpress to carry out their studies.

\section{REFERENCES}

[1] Smith, Aaron. Smartphone Ownership 2013. Pew Internet \& American Life Project, June 5, 2013,

http://pewinternet.org/Reports/2013/Smartphone-Ownership2013/Findings.aspx, accessed on December 8, 2013.

[2] Eagle, N., \& Pentland, A. (2006). Reality mining: sensing complex social systems. Personal and ubiquitous computing, 10(4), 255-268.

[3] Eagle, N., \& Pentland, A. (2005). Social serendipity: Mobilizing social software. Pervasive Computing, IEEE, 4(2), 28-34.

[4] Yun, T. J., \& Arriaga, R. I. (2013, April). A text message a day keeps the pulmonologist away. In Proceedings of the SIGCHI Conference on Human Factors in Computing Systems (pp. 1769-1778). ACM.

[5] Mendizabal, Enrique. For the 21st Century think tank: mobile data collection and research tools. On Think Tanks, May 9, 2011, http://onthinktanks.org/2011/05/09/for-the-21st-century-think-tankmobile-data-/collection-and-research-tools/, accessed on December 8, 2013
[6] Carter, S., Mankoff, J., \& Heer, J. (2007, April). Momento: support for situated ubicomp experimentation. In Proceedings of the SIGCHI conference on Human factors in computing systems (pp. 125-134). ACM.

[7] Rodgers, A., et al. "Do u smoke after txt? Results of a randomised trial of smoking cessation using mobile phone text messaging." Tobacco control, 14(4), 255-261.

[8] Franklin, V. L., Waller, A., Pagliari, C., \& Greene, S. A. (2006). A randomized controlled trial of Sweet Talk, a text-messaging system to support young people with diabetes. Diabetic Medicine, 23(12), 13321338.

[9] Free, C., et al. "Smoking cessation support delivered via mobile phone text messaging (txt2stop): a single-blind, randomised trial." The Lancet, 378(9785), 49-55.

[10] Ferrer-Roca, O., Cardenas, A., Diaz-Cardama, A., \& Pulido, P. (2004). Mobile phone text messaging in the management of diabetes. Journal of telemedicine and telecare, 10(5), 282-285.

[11] Neville, R., Greene, A., McLeod, J., Tracy, A., \& Surie, J. (2002). Mobile phone text messaging can help young people manage asthma. BMJ, 325(7364), 600 .

[12] Asiimwe, C., et al. (2011). Use of an innovative, affordable, and opensource short message service-based tool to monitor malaria in remote areas of Uganda. The American journal of tropical medicine and hygiene, 85(1), 26-33.

[13] Ngabo, F. et al. (2012). Designing and Implementing an Innovative SMS-based alert system (RapidSMS-MCH) to monitor pregnancy and reduce maternal and child deaths in Rwanda. The Pan African Medical Journal, 13.

[14] Crichton, R., Moodley, D., Pillay, A., Gakuba, R., \& Seebregts, C. J. (2013). An Architecture and Reference Implementation of an Open Health Information Mediator: Enabling Interoperability in the Rwandan Health Information Exchange. In Foundations of Health Information Engineering and Systems (pp. 87-104). Springer Berlin Heidelberg.

[15] Andrews, L., Russell-Bennett, R., \& Drennan, J. (2011). Capturing affective experiences using the SMS Experience Sampling (SMS-ES) method. International Journal of Market Research, 53(4), 1-27.

[16] Markett, C., Sánchez, I. A., Weber, S., \& Tangney, B. (2006). Using short message service to encourage interactivity in the classroom. Computers \& Education, 46(3), 280-293.

[17] Yun, T. J., et al. (2012, January). Using SMS to provide continuous assessment and improve health outcomes for children with asthma. In Proceedings of the 2nd ACM SIGHIT International Health Informatics Symposium (pp. 621-630). ACM. 